\title{
Erythematous Plaques on a Tattoo
}

Atieh Jibbe, MD; Vusala Snyder, MD; Garth Fraga, MD; Daniel Aires, MD; Anand Rajpara, MD

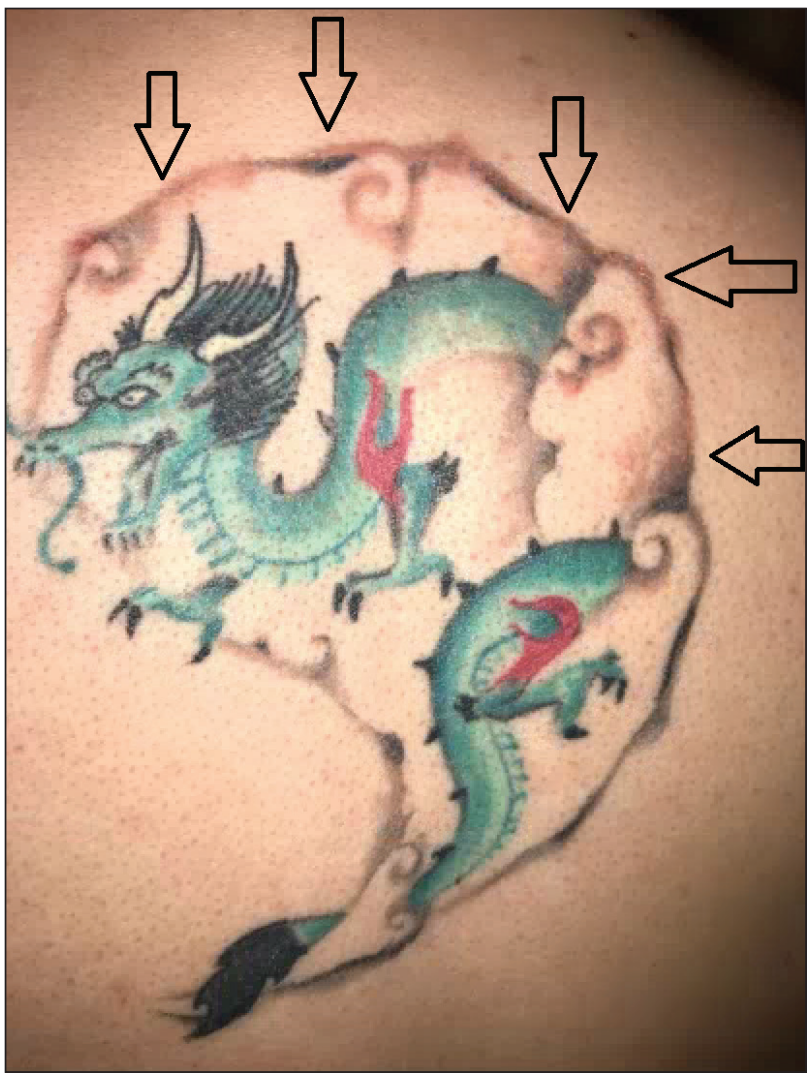

A 29-year-old man presented with increased redness, dryness, and pruritus at the periphery of a tattoo (arrows) on the upper back of 4 months' duration. He was diagnosed with human immunodeficiency virus 8 months prior to presentation and had a history of cystic fibrosis, eczema, and genital molluscum contagiosum. Laboratory analysis 1 month prior revealed a CD4 count of $42 \mathrm{cells} / \mathrm{mm}^{3}$ (reference range, 500-1200 cells $/ \mathrm{mm}^{3}$ ), and the viral load was 2388 copies $/ \mathrm{mL}$ (reference range, 20-10,000,000 copies/mL). Physical examination revealed multiple erythematous, eczematous, linear plaques along the dark gray lines of the tattoo. A $1.1 \times 0.7 \times 0.1-\mathrm{cm}$ shave biopsy specimen was obtained. After the biopsy, tretinoin cream $0.1 \%$ and betamethasone dipropionate ointment 0.05\% were prescribed to be alternately applied on the tattoo lesions until resolution.

\section{WHAT'S YOUR DIAGNOSIS?}

a. allergic contact dermatitis

b. epidermodysplasia verruciformis

c. pityriasis versicolor

d. sarcoidosis

e. squamous cell carcinoma

Drs. Jibbe, Fraga, Aires, and Rajpara are from the University of Kansas Medical Center, Kansas City. Drs. Jibbe, Aires, and Rajpara are from the Division of Dermatology, and Dr. Fraga is from the Division of Clinical Pathology. Dr. Snyder is from the University of Kansas School of Medicine. The authors report no conflict of interest.

Correspondence: Atieh Jibbe, MD, 3901 Rainbow Blvd, Mail Stop \#2025, Kansas City, KS 66160 (ajibbe@kumc.edu). 


\section{THE DIAGNOSIS:}

\section{Epidermodysplasia Verruciformis}

1 istopathologic examination demonstrated acanthosis and coarse hypergranulosis with enlarged keratinocytes exhibiting blue cytoplasmic discoloration (Figure), which was suggestive of acquired epidermodysplasia verruciformis (EV).

Acquired EV is a rare dermatologic condition associated with specific human papillomavirus (HPV) types that presents with recalcitrant lesions most commonly in the setting of immunosuppression. ${ }^{1}$ The most common HPV types associated with EV are HPV-5 and -8 , but associations with HPV-3, -9, -10, -12, -14, -15, $-17,-19$ to $-25,-36$ to $-38,-47$, and -50 also have been reported. ${ }^{1,2}$ Acquired EV has been identified in individuals with human immunodeficiency virus, as well as in immunosuppressed patients with organ transplantation, Hodgkin lymphoma, systemic lupus erythematosus, and IgM deficiency, and in patients taking immunosuppressive medications such as tumor necrosis factor $\alpha$ inhibitors. ${ }^{1,3}$ The diagnosis is clinicopathological with potential polymerase chain reaction studies to identify underlying HPV types.

Acquired EV presents as hypopigmented to red, tinea versicolor-like macules or as verrucous, flat-topped papules on the trunk, arms, and/or legs. ${ }^{4}$ Histopathology reveals viral epidermal cytopathic changes, blue cytoplasm, and coarse hypogranulosis. ${ }^{4}$

There is no standardized treatment regimen for acquired EV, and no single approach has proven to yield an efficacious clinical outcome. Topical treatment options include steroids, retinoids, immunomodulators, cryotherapy, and electrosurgery, whereas retinoids or interferon alfa have been used as oral systemic therapy. Photodynamic therapy also has been shown to improve symptoms. ${ }^{3}$ Combination therapy such as interferon alfa with zidovudine or imiquimod with oral isotretinoin has shown better results than any single treatment. ${ }^{4}$ Due to the underlying HPV infection and its role in promotion of skin cancer development, lesions can characteristically undergo malignant transformations into Bowen disease but most commonly invasive squamous cell carcinoma (SCC), with initial lesions preferentially affecting sunexposed areas due to the synergistic effect of UV light with EV-HPV lesions. The EV-HPV strains 5, 8, and 41 carry the highest oncogenic potential. ${ }^{5}$ Little is known of the true incidence of oncogenicity for acquired EV. Regardless, consistent sun protection and lifelong clinical examinations are critical for prognosis. ${ }^{5}$

The differential diagnosis of EV presenting in a tattoo includes allergic contact dermatitis, cutaneous sarcoidosis, pityriasis versicolor, and SCC. The pathology is critical to differentiate between these entities. The most frequently

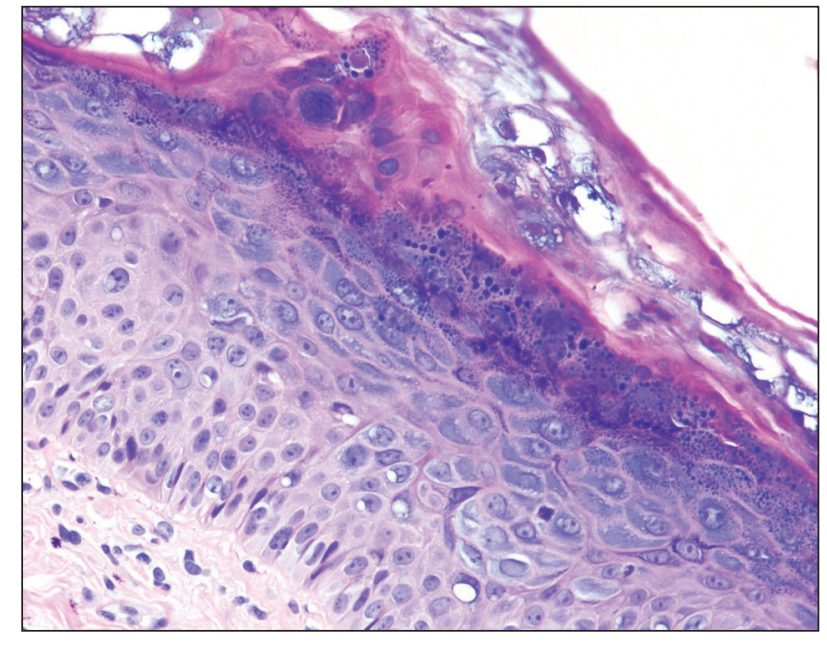

Epidermodysplasia verruciformis. Histopathologic examination demonstrated acanthosis and coarse hypergranulosis with enlarged keratinocytes exhibiting blue cytoplasmic discoloration (H\&E, original magnification $\times 40$ ).

reported skin reactions to tattoo ink include inflammatory diseases (eg, allergic contact dermatitis, granulomatous reaction) or infectious diseases (eg, bacterial, viral, fungal). ${ }^{6}$ Allergic contact dermatitis, typically red pigment, is a common tattoo reaction. The most common histologic feature, however, is spongiosis, which results from intercellular edema. It often is limited to the lower epidermis but may affect the upper layers if the reaction is severe. ${ }^{7}$ Cutaneous sarcoidosis is a great masquerader that can present in various ways; however, its salient features on pathology are noncaseating granuloma involving the basal cell layer and epithelioid granuloma consisting of Langerhans giant cells. ${ }^{8}$ Although pityriasis versicolor can present in young immunocompromised adults, histologically salient features are the presence of both spores and hyphae in the stratum corneum. ${ }^{9}$ Although immunosuppression is a known risk factor for SCC, it is characterized histologically by hyperkeratosis, parakeratosis, and acanthosis with thickened and elongated rete ridges. Scattered atypical cells and frequent mitoses are present. ${ }^{10}$

\section{REFERENCES}

1. Schultz B, Nguyen CV, Jacobson-Dunlop E. Acquired epidermodysplasia verruciformis in setting of tumor necrosis factor- $\alpha$ inhibitor therapy. J Am Acad Dermatol Case Rep. 2018;4:805-807.

2. DeVilliers EM, Fauquet C, Brocker TR, et al. Classification of papillomaviruses. Virology. 2004;324:17-27.

3. Zampetti A, Giurdanella F, Manco S, et al. Acquired epidermodysplasia verruciformis: a comprehensive review and a proposal for treatment. Dermatol Surg. 2013;39:974-980. 
PHOTO CHALLENGE DISCUSSION

4. Henley JK, Hossler EW. Acquired epidermodysplasia verruciformis occurring in a renal transplant recipient. Cutis. 2017;99:E9-E12.

5. Berk DR, Bruckner AL, Lu D. Epidermodysplasia verruciform-like lesions in an HIV patient. Dermatol Online J. 2009;15:1.

6. Napolitano M, Megna M, Cappello M, et al. Skin diseases and tattoos: a five-year experience. G Ital Dermatol Venereol. 2018;153:644-648.

7. Nixon RL, Mowed CM, Marks JG Jr. Allergic contact dermatitis. In: Bolognia J, Jorizzo JL, Schaffer JV, eds. Dermatology. 4th ed. Philadelphia, PA: Elsevier Saunders; 2018:242-259.
8. Ferringer T. Granulomatous and histiocytic diseases. In: Elston DM, Ferringer T, Ko C, et al, eds. Dermatopathology. 3rd ed. China: Elsevier; 2019:175-176.

9. Elewski BE, Hughey LC, Hunt KM, et al. Fungal diseases. In: Bolognia J, Jorizzo JL, Schaffer JV, eds. Dermatology. th ed. Philadelphia, PA: Elsevier Saunders; 2018:1329-1346.

10. Soyer HP, Rigel DS, McMeniman E. Actinic keratosis, basal cell carcinoma, and squamous cell carcinoma. In: Bolognia J, Jorizzo JL, Schaffer JV, eds. Dermatology. 4th ed. Philadelphia, PA: Elsevier Saunders; 2018:1887-1884.

WW.MDEDGE.COM/DERMATOLOGY

VOL. 105 NO. 6 I JUNE 2020 E37

Copyright Cutis 2020. No part of this publication may be reproduced, stored, or transmitted without the prior written permission of the Publisher. 